\title{
Half-edge Collapse Simplification Algorithm Based on Angle Feature ${ }^{1,2}$ JunFeng Li, ${ }^{2}$ YongBo Chen, ${ }^{3}$ MingSong Wang, ${ }^{3}$ GuoPing Wang \\ ${ }^{1}$ Guangdong University of Technology, GuangZhou, 510006, China; \\ ${ }^{2}$ Education training evaluation center of Guangdong Power Grid Co., Ltd, GuangZhou, 510650, China; \\ ${ }^{3}$ Beijing Kedong Electric Control System Co., Ltd, Beijing, 100192,China \\ huner2011@foxmail.com
}

Keywords: 3D model simplification, Half folding algorithm, Angle features

\begin{abstract}
A half folding algorithm based on angle features is proposed to meet the requirements of 3D model's simplification. The folded side with redundant data is determined by the side length and the angle between the side and the vertex's direction vector. The reserved vertex of the folded side is determined by the mean and the variance of the angle between the vertex's direction vector and all the sides through the vertex. The 3D model is simplified by folding the folded side to the reserved vertex. This algorithm is tested by Bunny 3D model, the result shows that this algorithm can reduce the redundant data without losing model features.
\end{abstract}

\section{Introduction}

Tree-dimensional model is the complicated geometry consisting of lots of vertexes and triangular patches. The larger the data sized of the three-dimensional model is, the more detailed the features of the model are. But larger data sized of model influences the analysis, storage and display of the model, especially complicated scenes with lots of three-dimensional models. In order to meet the requirements of real-time analyzing, storing and displaying three-dimensional model, before using three-dimensional model, there is need to simplify the model to reduce data capacity based on visual characteristics of model.

The researches about simplification algorithm of three-dimensional model have achieves some results. Hoppe proposed an edge collapse simplification algorithm based on energy equation, which simplifies three-dimensional model by introducing distance energy, representation energy and spring capacity. The algorithm has good simplification effect, but the calculated amount is large, which is difficult to meet the requirement of real-time simplification. Garland proposes an edge collapse simplification algorithm based on quadric error which determines the simplified vertex according to the quadratic sum of the distance from the vertex to the plane. The algorithm improves the simplification speed of the model, but the simplification effect is not good, especially when the surface of three-dimensional model has large fluctuation. Lu Guodong proposed a vertex deletion algorithm based on vector angle which deletes redundant vertexes according to the angle feature of the vertexes in the model. The algorithm can delete redundant vertexes of data, but it may have cavity after deleting vertexes.

The paper proposes half-edge collapse simplification algorithm based on angle feature which firstly uses the vector angle of side length of side to determine collapsed edges with data redundancy, and then uses the direction vector of vertexes on collapsed edges and the mean and the variance of angles of sides along the vertex to determine the simplified vertexes. And the three-dimensional model is simplified on the simplified vertexes. The execution speed of simplification algorithm is rapid, which can reduce data redundancy based on visual feature of model. 


\section{Edge Collapse Algorithm}

Edge collapse algorithm is a common three-dimensional simplification algorithm. It simplifies a side of three-dimensional model meeting the requirements into a vertex. And it connects all vertexes which are adjacent to two vertexes of the side and the simplified vertexes, and deletes the degraded vertexes and triangular patch. According to the difference of the position of the collapsed vertexes, edge-collapse algorithm can be divided into half-edge collapse algorithm and full-edge collapse algorithm. Half-collapse algorithm uses the original vertexes of three-dimensional mode as the simplified vertexes, and full-edge collapse algorithm uses new vertexes as simplified vertexes, as shown in Figure 1. Half-edge collapse algorithm not only can avoid the computation of the position of new vertexes, but also can reserve the normal and texture of original vertexes of three-dimensional model, which can't have extra memory access latency when data is stored, so the paper uses half-edge collapse simplification algorithm to simplify three-dimensional model. Half-edge collapse algorithm based on angle feature determines collapsed edges and simplified vertexes according to the angle feature of side and vertexes of three-dimensional model, and deletes data redundancy on the simplified vertexes.

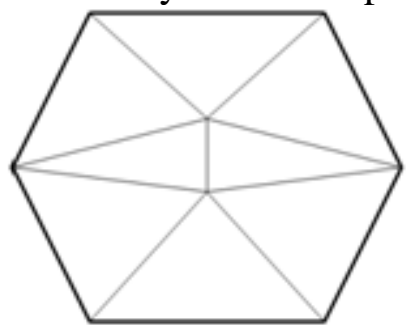

(a) Original grid

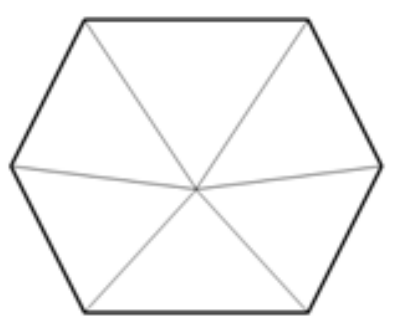

(b) Half-edge collapse algorithm

Figure 1 Edge collapse algorithm

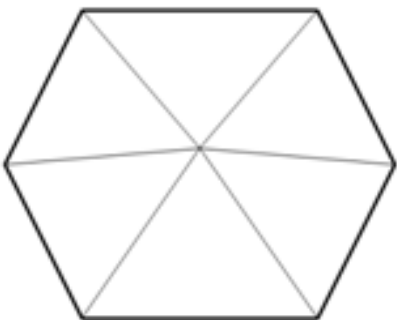

(c) Full-edge collapse algorithm

\section{Measure Metrics of Collapse Edges}

The three-dimensional model of modern equipment generally has the characteristics of complicated structure and large data size. Collapse edge measure metrics based on equidistant side length is difficult to fully reserve the visual feature of the model. In order to reduce data redundancy based on visual feature of the model, when the collapse edges are selected, the following factors need to be fully considered, vectorial angle of side length and side. Firstly, the shortest side is selected as collapse side. Then, the vectorial angle of the side is used to judge if the selected collapse edge is right.

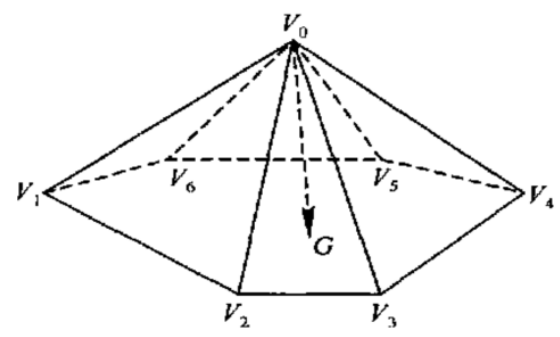

Fig. 2 Direction vector of vertex

Vectorial angle of edges. The direction vector of vertex is defined as the center of gravity of space polygon consisting of all adjacent vertexes. As shown in Figure 2, $\mathrm{G}$ means the space polygon consisting of all vertexes $V_{1} V_{2} V_{3} V_{4} V_{5} V_{6}$ that vertex $V_{0}$ is adjacent to $V_{0}$, and vector $\overline{V_{0} G}$ is the direction vector of vertex $V_{0}$.

The coordinate of the center of gravity of space polygon is defined as the mean of coordinates of all vertexes of space polygon. For the vertex $V_{0}$ of $n$ vertex vectors, the corresponding space coordinate of the center of gravity $\mathrm{G}$ is 


$$
\left(\frac{\sum_{0}^{n} x_{i}}{\mathrm{n}}, \frac{\sum_{0}^{n} y_{i}}{\mathrm{n}}, \frac{\sum_{0}^{n} z_{i}}{\mathrm{n}}\right)
$$

The direction vector $\overrightarrow{V_{0} G}$ of vertex $V_{0}$ is

$$
\left(\frac{\sum_{0}^{n} x_{i}}{n}-\mathrm{x}_{0}, \frac{\sum_{0}^{n} y_{i}}{\mathrm{n}}-\mathrm{y}_{0}, \frac{\sum_{0}^{n} z_{i}}{\mathrm{n}}-\mathrm{z}_{0}\right)
$$

The vector angle of sides is defined as the angle of the direction vector of a side and the side vertex, and each side has two vector angles. As shown in Fig. 2, $\angle V_{2} V_{0} G$ is the vector angle of side $V_{o} V_{2}$ at vertex $V_{o}$. According to the space coordinate of vertex and center of gravity in space polygon, two vector angles of each side can be computed.

Determining collapse edge. The shorter the distance of adjacent vertexes in three-dimensional model, the more similar the detail feature of two vertexes is, and the more possible the data redundancy is. The side length of triangular patch can be used to judge there is data redundancy near the side. In the inflection point and sharp edge of three-dimensional model, the side length of triangular patch is short, but the half-edge collapse may make model visual feature lose. In fact, the vector angle of side can reflect the smooth situation of the surface near the side. The larger the vector angle of side is, the more smooth the surface near the side. The smaller the vector angle of the side, the sharper the surface near the side. So the paper uses the vector angle of side length and side as the criterion of collapse edge. Firstly, the collapse edge to be collapsed is determined according to side length. Then, two vector angles of collapse edge are compared with the preset threshold to judge if the selected collapse edges are right.

Two vector angles of collapse edges are $\theta_{0}$ and $\theta_{1}$, and the preset threshold of vector angle of collapse edges is $\alpha$. When $\theta_{0}>\alpha$ and $\theta_{1}>\alpha$, the edge can be used as the collapse edge, or the edge can't be used as the collapse edge.

\section{Basic Flow of Algorithm}

The basic idea of half-edge collapse algorithm based on angle feature is as follows. Firstly, the vector angle of side length and side is used to determine the collapse edge to be collapsed. Then, the limitation of half-edge collapse is used to determine simplified vertex. Lastly, the collapse edge is collapsed at the simplified vertex, and the degraded vertex and triangular patch is deleted. The steps are as follows.

According to the space coordinate of vertex in three-dimensional model, the side length of adjacent vertexes is computed. Then, the side length is ordered to establish side length list.

The shortest side length is selected from side length list as collapse edge, and two vector angles of the side is computed.

Two vector angles of collapse edge is compared with the preset threshold. If two vector angles are larger than the preset threshold, the selection of collapse side is right, or the side is deleted in side length list, and then turning to step 2. And the collapse side is selected again.

According to formula (3) and formula (4), the mean and variance of two vertex angles of collapse edge can be achieved.

The mean and variance of two vertex angles of collapse edge is substituted into formula (5), and

is compared with the preset threshold ${ }^{\alpha_{1}}$ and ${ }^{\alpha_{2}}$. The vertexes meeting the collapse restriction are selected as simplified vertex. If two vertexes of collapse edge meet collapse limitation, the vertex with small angle mean is selected as simplified vertex. If two vertexes of collapse edge can't meet the collapse restriction, the side is deleted from side list length, and turning to Step 2. And the collapse edge is selected again. 
Half-edge collapse is made to connect all vertexes with simplified vertexes, and the degraded vertexes and triangular patch are deleted.

The collapse edge is deleted in side length list, and turning to step 2 to select collapse edge again until the simplification requirements are met.

\section{Experiment Results}

The algorithm proposed in the paper is implemented on HP PC by using $\mathrm{VC}++$ programming. And Bunny three-dimensional data mode provided Stanford University is used to make experimental verification on the algorithm.

In the experiment, the preset threshold of vector angle of collapse edge $\alpha=60^{\circ}$, and the threshold of mean of vertex angle is $\alpha_{1}=45^{\circ}$. And the preset threshold of the variance of vertex angle is $\alpha_{2}=5.5$. The algorithm in the paper is compared with quadratic error algorithm, and the simplified effect is shown in Figure 3. And the comparison of execution time between two algorithms is shown in Table 1.

From Figure 3, we can see that when the algorithm in the paper and quadratic error algorithm is used to simplify the Bunny original model into the same proportion $60 \%$, the visual feature of the algorithm in the paper is closer to the original model, especially in the eye and leg area of model surface fluctuation. From Table 1, we can see that the execution time of the algorithm in the paper is longer than that of quadratic error algorithm, the reason for which is that the algorithm in the paper needs to make extra angle computation. But the algorithm in the paper also can meet the real-time simplification requirement of three-dimensional model. The experimental results indicate that half-edge collapse algorithm based on angle feature not only has rapid execution speed, but also can reduce data redundancy based on ensuring model visual feature.
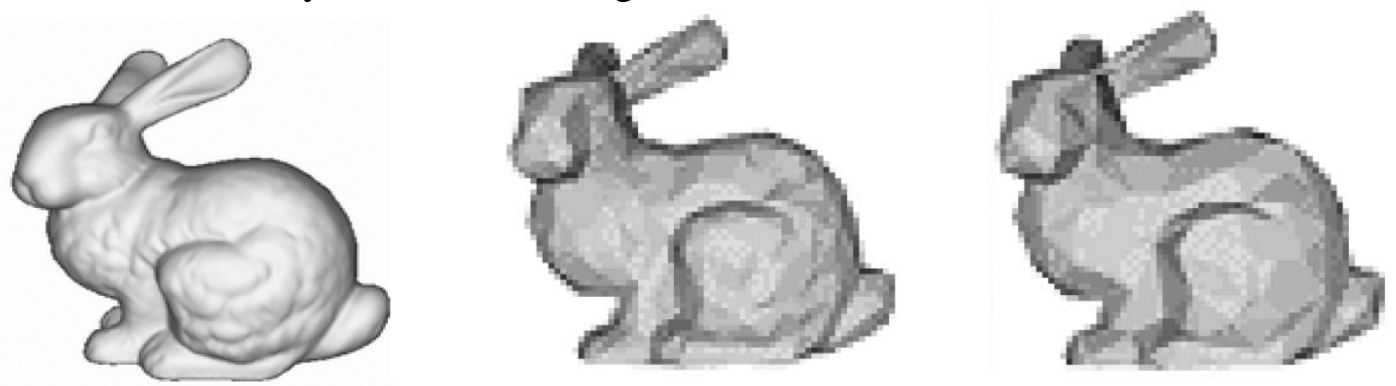

(a) Bunny original model (b) Effect of using the algorithm in the paper to simplify 60\%(c) Effect of using quadratic error algorithm to simplify $60 \%$

Fig. 3 Simplification effect of the algorithm in the paper and quadratic error algorithm

Table 1 Comparison of execution time of the algorithm in the paper and quadratic error algorithm

\begin{tabular}{|c|c|c|c|c|c|c|}
\hline & \multicolumn{3}{|c|}{ Algorithm in the paper } & \multicolumn{3}{c|}{ Quadratic error algorithm } \\
\hline Model & $30 \%$ & $60 \%$ & $90 \%$ & $30 \%$ & $60 \%$ & $90 \%$ \\
\hline Bunny & $3.58 \mathrm{~s}$ & $5.44 \mathrm{~s}$ & $7.02 \mathrm{~s}$ & $2.94 \mathrm{~s}$ & $4.12 \mathrm{~s}$ & $5.24 \mathrm{~s}$ \\
\hline
\end{tabular}

\section{Conclusion}

Three-dimensional model simplification algorithm has the problems that execution time is long and simplification effect is not ideal, for which the paper proposes half-edge collapse simplification algorithm based on angle feature which uses the vector angle of side length and side to determine the collapse edge with data redundancy, and uses the mean and variance of angle between direction vector of vertex and sides along the vertex to determine the simplified vertex. The experiment results indicate that the execution speed of the algorithm not only is rapid, but also can reduce data redundancy based on ensuring model visual feature. 


\section{References}

[1] Q.P. Zhao, Overview of virtual reality, Chinese, 2009, 39(1): 2-46

[2] R. Wang, J.S. Li, G.Q. Liu, Three-dimensional Geometry Model Simplification Algorithm Keeping Visual Feature, 2008,35(7): 63-68.

[3] G.D. Lu, P. Xu, X. Wen, Triangular Grid model simplification algorithm based on angle vector, Journal of Engineering Design, 2005,12(2):124-128.

[4] Kalvin A, Taylor R. Superfaces: Ploygonal mesh simplification with bounded error. IEEE Computer Graphics and Applications, 1996, 16(3): 64-77.

[5] Schroeder W J, Zarge J A et al. Decimation of triangle meshes. Computer Graphics, 1992, 26(2): 65-70

[6] Hoppe H, Dc Rose T. Mesh optimization. Computer Graphics, 1993,27(1): 19-26 